\section{POSITRON ANNIHILATION}

\section{Industrial Applications Develop}

Positrons are often associated with high-energy physics and accelerators, and hence are thought to occupy the realm of basic science. Less well known is the important role they play in materials science after it was discovered in the second half of the 1960s that the results of positron annihilation experiments in solids are strongly dependent on the history of the sample [Schultz P.J. \& Lynn K.G., Rev. Mod. Phys. 60 (1988) 701]. Following some 25 years of research and development in the laboratory, positron annihilation is now finding its way into industry. This was illustrated by the first Europhysics Industrial Workshop (EIW) on Industrial Applications of Positron Annihilation (10-12 March 1994), where contributions could roughly be divided into three categories: defects in metals, defects in semiconductor materials and devices, and industrial applications of positron tomography.

\section{Metals}

Metals are the first materials in which positrons were used to study defects, and where much of the methodology has been developed. The field has become mature, as illustrated by a portable system for the non-destructive testing of metals based on Doppler broadening $S$ parameter measurements (see insert for a description of experimental techniques). Developed by M.T. Hutchings of AEA Technology, Harwell, it is designed to signal the early stages of

\section{Two Principal Techniques}

There are, grossly speaking, two different modes in which positrons are used to provide information on defects in materials. First of all, upon analysis, the lifetime spectrum of positrons in a material may be shown to contain several lifetime components, reflecting the various channels via which positrons decay. For example, positrons may annihilate from the bulk through the formation of positronium (Ps) atoms either in a void or at an interface after being trapped in various types of defects. When the sample contains open-volume defects such as vacancies or voids, the positron lifetime spectrum shows a clearly resolved long-lived component. This reflects the below-average density of electrons "seen" by the positron since it is trapped in a defect. In general, the lifetime of a positron in a defect depends on the size of the open volume of the defect.

Similarly, the momentum density of a positron-electron pair trapped in a defect shows a relatively large component at low momenta, in agreement with the fact that a vacancy implies the absence of an atom with its bound electrons. This difference can be seen by measuring the Doppler broadening of the $511 \mathrm{keV}$ annihilation line and observing the variations of the fractions $S$ and $W$ of positrons annihilating with low momentum (mainly valence) electrons and high momentum (mainly core) electrons, respectively. When compared with the results of measurements in a carefully annealed sample, the relative lack of high electronic momenta in a defect is reflected in a smaller amount of Doppler broadening of the $511 \mathrm{keV}$ line, and hence a higher value of the $S$ parameter and a lower value of the $W$ parameter.

The $S$ and $W$ parameters can be used in depth-profiling measurements in which the damage due to mechanical stress, fatigue, creep or radiation damage since the $S$ parameter saturates at $\approx 10 \%$ of fatigue life. Another instrument for use in the field, developed by a collaboration between universities in Saarbrücken and Halle, employs the lifetime of positrons to study fatigue. Damage was found even after the very first elastic cycle in materials which were well annealed at the outset. Other interesting applications of positron methods in physical metallurgy concern the monitoring of em-

energy $E$ of a monochromatic beam of slow positrons is varied between say $100 \mathrm{eV}$ and $50 \mathrm{keV}$ and the $S$ or $W$ parameter is measured as a function of $E$. Since the positron energy determines the depth at which the positrons are implanted in the material, this provides a method of measuring defect type

\section{Precision Depth Profiling}

When a monoenergetic beam of slow positrons strikes a sample, the positrons penetrate into the material and reach thermal equilibrium with the lattice in a period which is two orders of magnitude shorter than the positron lifetime. The implantation takes place according to a profile which peaks at a depth which depends on the initial energy of the positrons. After thermalization, the positrons diffuse through the material over a distance given by the diffusion length, which depends on the perfection of the material. In perfectly crystalline $\mathrm{Si}$, the diffusion length is $\approx 2500 \AA$ while in $\mathrm{Si}$ with a high concentration of defects it can be as small as $20 \AA$. In this way it is possible to deposit the positrons approximately at a pre-selected depth which increases with the positron energy.

The figure shows the $S$ parameter (the fraction of positrons annihilating with low momentum electrons) as a function of positron energy for a layer of hydrogenated amorphous silicon grown on a silicon substrate. The high values of the parameter at low positron energies indicate a large concentration of voids in the deposited layer. The void concentration decreases with increasing deposition temperature. As the positron energy increases, a larger fraction of the positrons diffuses into the substrate and the $S$ parameter tends to the value for the crystalline substrate. saturates. brittlement of reactor vessel steels under the effect of neutron irradiation.

\section{Semiconductors}

A special feature of semiconductors is the occurrence of charged defects owing to localized electronic states in the band gap. Neutral or negatively charged defects can act as traps or bind a positron in a Rydberg state which extends over many lattice spacings; positively charged defects do not trap positrons and are therefore not observed.

In Si-based very-large-scale-integrated (VLSI) semiconductor devices it becomes increasingly important to characterize defects and control their concentration as the size of the device and the lateral dimensions of the conducting lines become smaller. The very high sensitivity of positrons to openvolume defects makes it possible to demonstrate the presence of defects in materials which according to the usual methods of characterization are "defect-free". These defects can originate in the low-temperature molecular beam epitaxy process with which the layers are grown, or be the result of radiation damage during $\mathrm{X}$-ray lithography needed to produce detail on a scale of less than $0.25 \mu \mathrm{m}$. Defects in the semiconductor

and concentration in a depth-dependent way (see insert).

The sensitivity of the method is high: monovacancy concentrations of $10^{-6}$ per atom can be measured. At concentrations greater than $10^{-3}$ per atom, the trapping
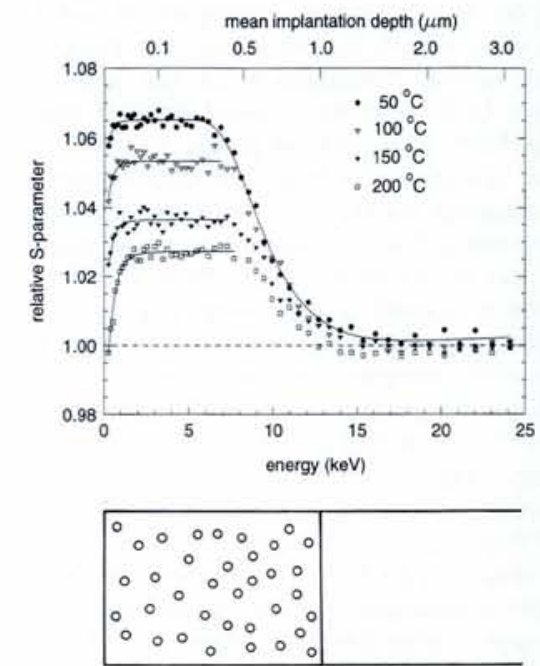

Positron-annihilation depth-profiling measurements of a $1 \mu \mathrm{m}$ thick hydrogenated amorphous silicon layer for solar-cell applications grown on a crystalline silicon substrate at different temperatures using a plasma-enhanced chemical vapour deposition method. a, upper) Experimental data and the results of modelling calculations (lines). b, lower) Schematic illustration showing the distribution of voids in a cross-section through the silicon layer. 
material affect the electrical properties of the device, as is shown by the different electrical characteristics of Schottky diodes made from material which has been found using positrons to be defect-free or not. Defects in the metallic lines on the semiconducting substrate are also important since they can coalesce into larger clusters and even voids following heating under operating conditions. A series of voids may then lead to rupture of the line, resulting in device failure.
Another region where defects are formed is at the $\mathrm{S}_{i} \mathrm{O}_{2} / \mathrm{Al}(\mathrm{Cu})$ interface. These defects, which result in contact peeling, cannot be observed by transmission electron microscopy, but are easily detected with the aid of positron depth-profiling. Electromigration probably plays a role, since the results depend on the time that current has been passed through the device.

In compound semiconductor materials, there are new types of defects in addition to the ones encountered in homogeneous semiconductors (they include vacancies on the different sub-lattices and atoms sitting on the wrong sublattice). Several results concerning technologically significant GaAs and GaAlAs materials were reported at the EIW in invited talks by P. Hautojärvi (Helsinki University of Technology) and M.R. Brozel (Manchester Institute of Science and Technology). Positron annihilation is particularly successful in identifying defects such

\section{ab initio Calculations Characterize Defects}

The proper functioning of semiconducting devices hinges on characterizing structural defects which modify electrica and optical properties. This need has triggered considerable effort using many different experimental techniques, including recent theoretical [1] and experimental [2] work based on employing positrons as probes. However, in relating experimenta observations to theoretical results, traditional band-structure calculations have difficulty in describing how electrons in the bulk material behave in the vicinity of an empty lattice site because they rely on the periodicity of the lattice, and often assume that the ionic configurations are known. The problem is more complicated if a positron is trapped in a vacant site because the atomic relaxations are modified by the presence of the positron. This effect has to be included in the calculations if one is to make comparisons with measurements of the angular correlation of the annihilation radiation (the so-called ACAR technique) which provide fingerprints of different kinds of defects.

Quantum molecular dynamics has been used [1] to perform ab initio studies of positron trapping at a vacancy site. The CarParrinello (CP) scheme [3] for the simultaneous optimization of the atomic and electronic structures has been extended to include the positronic degrees of freedom on the basis of a two-component densityfunctional theory [4]. Calculations have been performed for the singly negative As vacancy in GaAs which include self-consistently the lattice relaxations induced by the presence of both the defect and the positron. The structural, electronic and positronic characteristics have therefore been obtained without a priori assumptions concerning the symmetry of the vacancy or the positron wavefunction.

It has been found that, in the presence of a positron, the volume of the vacancy increases and its symmetry is lowered. The wavefunction of the positron is found to be well localized (this is shown schematically in the left part of the figure which compares the delocalized wavefunction of a positron in the bulk with the localized wavefunction at an As vacancy). Moreover, the results of these $a b$ initio calculations provide positron life-
Positron Wavefunction Experiment Theory
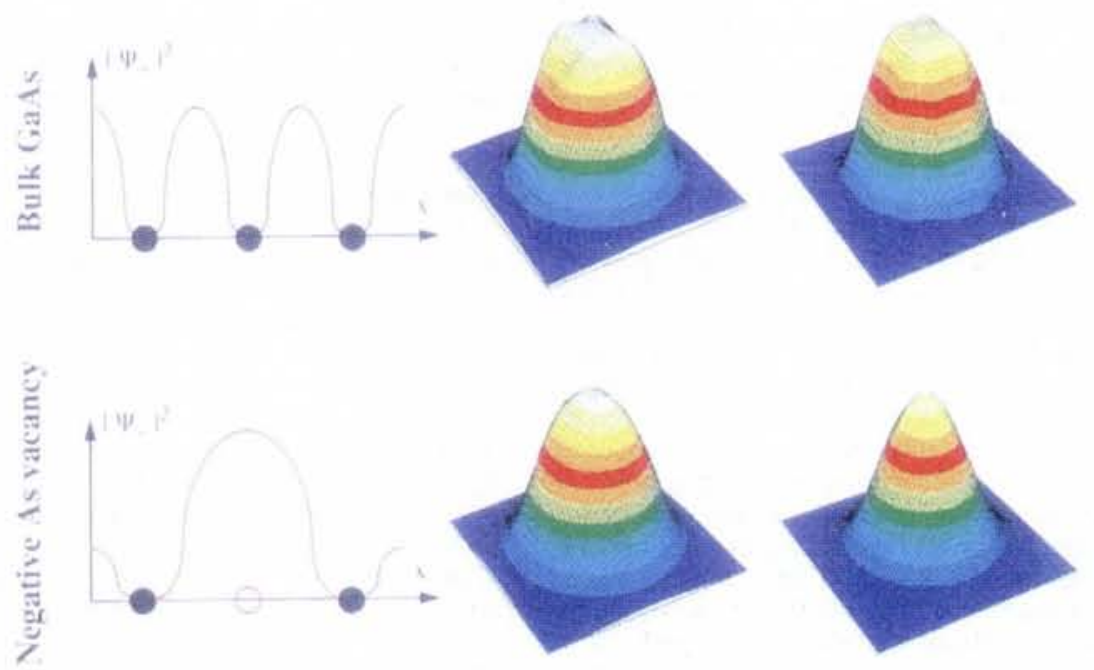

Electron momentum distributions in GaAs as seen by positrons (centre and right). Being positively charged, positrons in solids are either confined to the interstitial region of the lattice or trapped by defects. Note the excellent agreement between the experimental and calculated ACAR distributions for annihilation in bulk GaAs (upper) and singly negative As vacancies (lower). The momentum distributions are sensitive to the site of annihilation, thus offering a powerful tool to investigate defects in technologically significant materials. Positron wavefunctions are illustrated schematically on the left to demonstrate delocalization at a vacancy.

times in good agreement with experimental values [5]

More precise information on the behaviour of electrons in the vicinity of an empty lattice site requires two-dimensional ACAR measurements. This method [6] provides maps of the electron momentum distribution, as seen by the positrons. Initially applied to investigate Fermi surfaces in metals and superconductors, it has been used [2] to measure the electron-positron momentum distribution in bulk GaAs and at various kinds of vacancies and antisites.

For the bulk and the singly negative As vacancy, there is a good agreement between the ACAR profiles and distributions calculated using the CP approach (see the right hand-side of the figure). One notes that the shapes of ACAR distributions are quite different for the bulk and the vacancy, outlining the sensitivity of ACAR measurements and the precision of the CP-calculations. The results are very encouraging as they open a new way to investigate and characterize defects in many semiconductors.

Using samples carefully characterized by positron lifetime and Doppler broadening measurements, ACAR maps have been obtained for antisites and the As and $\mathrm{Ga}$ vacancies in various negatively and neutrally charged states. The measurements show that each type of structural defect in GaAs has a characteristic ACAR distribution which depends on the charge state of the defect. This information could possibly be used in the future to design a tool capable of identifying and quantifying defects in industrial materials. The method looks promising for GaAs and could be extended to other elemental and compound semiconductors. In appreciating the opportunities, several groups worldwide have started to work along these lines; the EIW workshop offered an unique occasion to present some of the new developments.

A.A. Manuel, Geneva University

[1] Giligen L. et al., Phys. Rev. Lett. 72 (1994) 3214.

[2] Ambigapathy R. et al., Phys. Rev. B (1994), to be published.

[3] Car R. \& Parrinello M., Phys. Rev. Lett. 55 (1985) 2471

[4] Boronski E. \& Nieminen R.M., Phys. Rev. B 34 (1986) 3820 .

[S] Saarinen K. et al., Phys. Rev. B 44 (1991) 10585.

[6] Peter M., IBM J. of Res. \& Devel. 33 (1989) 3. 
as negative vacancies associated with the metastable state of the native mid-gap donor, the so-called EL2 centres which control compensating mechanisms in semi-insulating GaAs (see figure). The technique is able to show that the open volume of these vacancies is smaller than that of Ga or As vacancies, strongly supporting recent models which propose a [111]oriented vacancy-interstitial complex for the structures of these centres. Furthermore, As vacancies seem to play an important role as recombination (RC) centres in GaAs since positron annihilation has demonstrated that their concentration is correlated with that of RC centres. The latter are detected by infra-red absorption and have been shown to control the minority carrier lifetime in bulk semiinsulating GaAs.

Proof that the characterisation of defects in semiconductors by positron annihilation is becoming more firmly rooted also stems from interesting studies by a group based in Geneva and Lausanne of the negatively charged As vacancy in doped GaAs. By combining Car/Parrinello-type ab initio electronic structure/molecular dynamics calculations with measurements of the momentum density of electron-positron pairs by observing the two-dimensional angular correlation between the $511 \mathrm{keV}$ annihilation quanta (the so-called two-dimensional ACAR technique), it can be established that the atoms around the vacancy relax in an outward direction when a positron is trapped in the vacancy (see insert). This type of study is important for understanding the behaviour of positrons in defects.

A simple but powerful extension of the Doppler broadening method has been

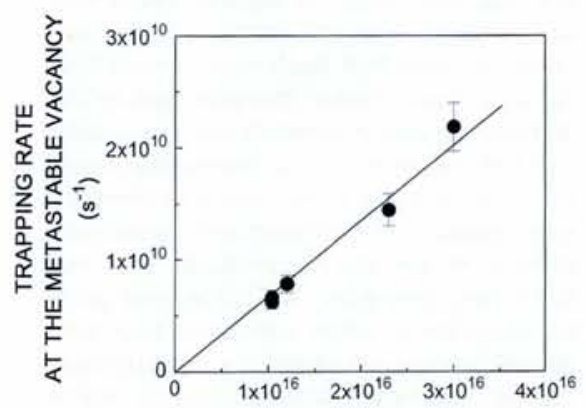

TOTAL EL.2 CONCENTRATION $\left(\mathrm{cm}^{-3}\right)$

Positron annihilation is particularly successful in identifying negative vacancies associated with the metastable state of the EL2 centre in $\mathrm{GaAs}$. The figure gives the positron trapping rate at the metastable vacancies generated when EL2 centres are excited to their metastable state. The data are plotted as a function of the EL2 concentration determined from Fourier transform infra-red absorption in bulk GaAs crystals from various suppliers [C. Le Berre, Université d'Orsay, Paris XI (1994), unpublished]. The data strongly support the model of a (111)-oriented vacancy-interstitial complex for the structure of these centres [Krause R. et al., Phys. Rev. Lett. 65 (1990) 3329].

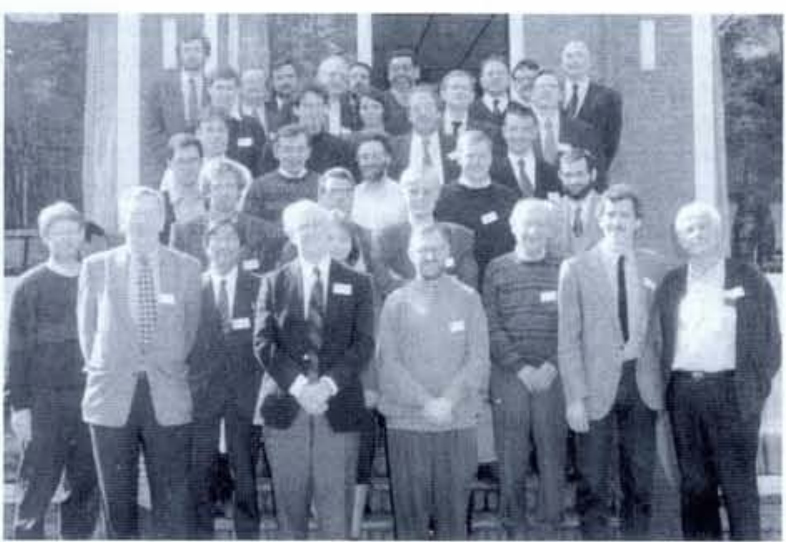

The participants at the first Europhysics Industrial Workshop (EIW) on Industrial Applications of Positron Annihilation (10-12 March 1994) which was held in a secluded hotel near the picturesque village of Oisterwijk in the south of The Netherlands. EIWs aim to bring together scientists from universities and from industry in order to promote the application of new physical methods to industrial problems. The workshop, the 12th in the series of EIWs and chaired by A. van Veen (IRI, Delft) and C. Corbel (Saclay), attracted some 40 scientists from both secors. The proceedings of the workshop will be published as a upplement to the Journal de Physique.

shown to allow one to distinguish between various kinds of defects. It is actually a revival of an old idea [Mantl S. \& W. Triftshäuser W., Phys. Rev. B 17 (1978) 1645] and consists of simultaneously examining the variations of the $S$ and $W$ parameters. It has been shown for CdTe and that the data points representing samples with different concentrations of a specific type of defect (e.g., monovacancies) fall on a straight line in a $S$ versus $W$ plot, while samples containing a different type of defect (say divacancies) define a different straight line in the same plot. This represents a powerful method of defect identification. Furthermore, it has been demonstrated in $\mathrm{Cd}_{(1-x)} \mathrm{Te}_{x} \mathrm{Hg}$ that positrons can be used to discriminate ion-type acceptors from vacancy-type acceptors and to determine the concentration of each type.

\section{Other Materials, Large Scales}

Reports at the workshop about depth profiling in diamond-like coatings, magnetic multilayers and layers of paint show that the application of positron annihilation is not limited to metals and semiconductors. Nor are positron methods restricted to the microscopic realm, as is witnessed by work on positron emission tomography at Shell that was reported by $\mathrm{G}$. Jonkers. With the aid of gamma-ray cameras and positron emitting tracers it is possible to study processes as diverse as the flooding of oilfilled reservoir rock with water, the movement of particles in a rotating drum and the oil flow in engines while they are running. The value of the use of positron annihilation is the feasibility of safe in situ imaging of complicated processes without disturbing them.

P.J. Mijnarends, IRI, Delft

\section{POSITRON FACILITIES}

\section{Facilities-based Capabilities Increase}

W. Triftshäuser from the Institut für Nukleare Festkörperphysik, Universität der Bundeswehr, Munich, described at the Europhysics Industrial Workshop Industrial Applications of Positron Annihilation Europe's move towards larger and more sophisticated facilties for positron annihilation.

Many groups are using positrons from radioactive sources in lab-scale devices to tackle a variety of applications (see Directory of Positron Groups in Europe, p. 181). A few, together with at least one commercial company, have developed equipment to measure the angular correlation of positron annhilation radiation which gives detailed information on the electron-positron momentum density, especially if positron-sensitive detectors are used. However, opportunities are limited since the single crystal sample must be fairly large (about the same size as the 100-200 $\mu \mathrm{m}$ depth to which positrons from radioactive sources penetrate into the sample). It is clearly advantageous to have available high intensity, narrow beams capable of probing small samples. So there is a trend towards dedicated positron sources operating as user facilities that offer monoenergetic continuous beams of variable energy and of high intensity. At the same time, pulsed systems originally designed for surface studies are being developed as scanning microscopes by reducing considerably the beam size.

\section{Continuous Beams}

The application of "conventional" low-energy continuous positron beams has increased significantly these last few years owing to the development of more efficient moderators to give beams with a narrower distribution of energy. Positrons from primary radioactive sources are passed into a moderator acting as a secondary source of low-energy positrons and the low- and high-energy positrons are then separated and guided electrically and/or magnetically to the target.

However, the intensity of radioisotope source-based beams are limited by selfabsorption. One approach to increase the intensity is based on pair production from Bremsstrahlung in a linac, but heating and cooling of the target foil used to produce the positrons impose serious limitations. Another disadvantage is that most linacs are pulsed so the positron beam is also pulsed. A group at the University of Gent uses a specially designed Penning trap to generate a semicontinuous beam of some $4 \times 10^{7}$ positrons/s by smearing out pairs of pulses. 